\title{
ON EULERIAN EQUILIBRIA IN $K$-ORDER APPROXIMATION OF THE GYROSTAT IN THE THREE-BODY PROBLEM
}

\author{
J. A. VERA AND A. VIGUERAS \\ Received 9 June 2006; Revised 21 September 2006; Accepted 9 November 2006
}

We consider the noncanonical Hamiltonian dynamics of a gyrostat in the three-body problem. By means of geometric-mechanics methods we study the approximate Poisson dynamics that arises when we develop the potential in series of Legendre and truncate this in an arbitrary order $k$. Working in the reduced problem, the existence and number of equilibria, that we denominate of Euler type in analogy with classic results on the topic, are considered. Necessary and sufficient conditions for their existence in an approximate dynamics of order $k$ are obtained and we give explicit expressions of these equilibria, useful for the later study of the stability of the same ones. A complete study of the number of Eulerian equilibria is made in approximate dynamics of orders zero and one. We obtain the main result of this work, the number of Eulerian equilibria in an approximate dynamics of order $k$ for $k \geq 1$ is independent of the order of truncation of the potential if the gyrostat $S_{0}$ is close to the sphere. The instability of Eulerian equilibria is proven for any approximate dynamics if the gyrostat is close to the sphere. In this way, we generalize the classical results on equilibria of the three-body problem and many of those obtained by other authors using more classic techniques for the case of rigid bodies.

Copyright ( 2006 J. A. Vera and A. Vigueras. This is an open access article distributed under the Creative Commons Attribution License, which permits unrestricted use, distribution, and reproduction in any medium, provided the original work is properly cited.

\section{Introduction}

In the study of configurations of relative equilibria by differential geometry methods or by more classical ones, we will mention here the papers of Wang et al. [8], about the problem of a rigid body in a central Newtonian field; Maciejewski [3], about the problem of two rigid bodies in mutual Newtonian attraction. These papers have been generalized to the case of a gyrostat by Mondejar and Vigueras [4] to the case of two gyrostats in mutual Newtonian attraction. 
For the problem of three rigid bodies we would like to mention that Vidyakin [7] and Dubochine [1] proved the existence of Euler and Lagrange configurations of equilibria when the bodies possess symmetries; Zhuravlev and Petrutskii [9] made a review of the results up to 1990 .

In Vera [5] and a recent paper of Vera and Vigueras [6] we study the noncanonical Hamiltonian dynamics of $n+1$ bodies in Newtonian attraction, where $n$ of them are rigid bodies with spherical distribution of mass or material points and the other one is a triaxial gyrostat.

Let us remember that a gyrostat is a mechanical system $S$, composed of a rigid body $S^{\prime}$, and other bodies $S^{\prime \prime}$ (deformable or rigid) connected to it, in such a way that their relative motion with respect to its rigid part do not change the distribution of mass of the total system $S$, (see Leimanis [2] for details).

In this paper, we take $n=2$ and as a first approach to the qualitative study of this system, we describe the approximate dynamics that arises in a natural way when we take the Legendre development of the potential function and truncate this until an arbitrary order. We give global conditions on the existence of relative equilibria and in analogy with classic results on the topic, we study the existence of relative equilibria that we will denominate of Euler type in the case in which $S_{1}, S_{2}$ are spherical or punctual bodies and $S_{0}$ is a gyrostat. Necessary and sufficient conditions for their existence in a approximate dynamics of order $k$ are obtained and we give explicit expressions of these equilibria, useful for the later study of the stability of the same ones. A complete study of the number of Eulerian equilibria is made in approximate dynamics of orders zero and one. The number of Eulerian equilibria in an approximate dynamics of order $k$ for $k>1$ is independent of the order of truncation of the potential if the gyrostat $S_{0}$ is close to the sphere. The instability of Eulerian equilibria is proven for any approximate dynamics if the gyrostat is close to the sphere. The analysis is done in vectorial form avoiding the use of canonical variables and the tedious expressions associated with them.

We should notice that the studied system has potential interest both in astrodynamics (dealing with spacecraft) as well as in the understanding of the evolution of planetary systems recently found (and more to appear), where some of the planets may be modeled like a gyrostat rather than a rigid body. In fact, the equilibria reported might be well compared with the ones taken for the "parking areas" of the space missions (GENESIS, SOHO, DARWIN, etc.) around the Eulerian points of the Sun-Earth and the Earth-Moon systems.

To finish this introduction, we describe the structure of the article. The paper is organized in five sections, two appendices, and the bibliography. In these sections we study the equations of motion, Casimir function and integrals of the system, the relative equilibria and the existence of Eulerian equilibria in an approximate dynamics of order $k$, in particular the study of the bifurcations of Eulerian equilibria in an approximate dynamics of orders zero and one.

\section{Equations of motion}

Following the line of Vera and Vigueras [6] let $S_{0}$ be a gyrostat of mass $m_{0}$ and $S_{1}, S_{2}$ two spherical rigid bodies of masses $m_{1}$ and $m_{2}$. We use the following notation. 


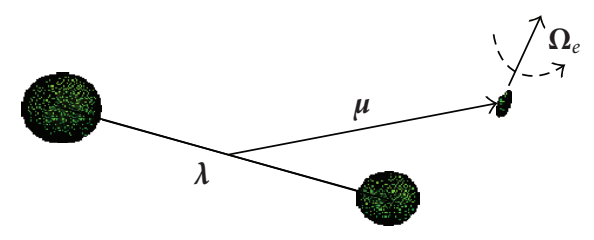

Figure 2.1. Gyrostat in the three-body problem.

For $\mathbf{u}, \mathbf{v} \in \mathbb{R}^{3}, \mathbf{u} \cdot \mathbf{v}$ is the dot product, $|\mathbf{u}|$ is the Euclidean norm of the vector $\mathbf{u}$, and $\mathbf{u} \times \mathbf{v}$ is the cross-product. $\mathbf{I}_{\mathbb{R}^{3}}$ is the identity matrix and $\mathbf{0}$ is the zero matrix of order three. Let $\mathbf{z}=\left(\boldsymbol{\Pi}, \boldsymbol{\lambda}, \mathbf{p}_{\lambda}, \boldsymbol{\mu}, \mathbf{p}_{\mu}\right) \in \mathbb{R}^{15}$ be a generic element of the twice reduced problem obtained using the symmetries of the system, where $\Pi=\square \Omega+\mathbf{l}_{r}$ is the total rotational angular momentum vector of the gyrostat, $\square=\operatorname{diag}(A, B, C)$ is the diagonal tensor of inertia of the gyrostat, and $\boldsymbol{\Omega}$ is the angular velocity of $S_{0}$ in the body frame, $\mathfrak{J}$, which is attached to its rigid part and whose axes have the direction of the principal axes of inertia of $S_{0}$. The vector $\mathbf{l}_{r}$ is the gyrostatic momentum that we suppose constant and is given by $\mathbf{l}_{r}=$ $(0,0, l)$. The elements $\lambda, \boldsymbol{\mu}, \mathbf{p}_{\lambda}$, and $\mathbf{p}_{\mu}$ are, respectively, the barycentric coordinates and the linear momenta expressed in the body frame $\mathfrak{J}$ (see Figure 2.1).

The twice reduced Hamiltonian of the system, obtained by the action of the group $\mathrm{SE}(3)$, has the following expression:

$$
\mathscr{H}(\mathbf{z})=\frac{\left|\mathbf{p}_{\boldsymbol{\lambda}}\right|^{2}}{2 g_{1}}+\frac{\left|\mathbf{p}_{\boldsymbol{\mu}}\right|^{2}}{2 g_{2}}+\frac{1}{2} \Pi^{-1} \Pi-\mathbf{l}_{r} \cdot \square^{-1} \Pi+\mathscr{V}
$$

with

$$
\begin{gathered}
M_{2}=m_{1}+m_{2}, \quad M_{1}=m_{1}+m_{2}+m_{0}, \\
g_{1}=\frac{m_{1} m_{2}}{M_{2}}, \quad g_{2}=\frac{m_{0} M_{2}}{M_{1}},
\end{gathered}
$$

with $\mathscr{V}$ being the potential function of the system given by the formula

$$
\mathscr{V}(\boldsymbol{\lambda}, \boldsymbol{\mu})=-\left(\frac{G m_{1} m_{2}}{|\boldsymbol{\lambda}|}+\int_{S_{0}} \frac{G m_{1} d m(\mathbf{Q})}{\left|\mathbf{Q}+\boldsymbol{\mu}+\left(m_{2} / M_{2}\right) \boldsymbol{\lambda}\right|}+\int_{S_{0}} \frac{G m_{2} d m(\mathbf{Q})}{\left|\mathbf{Q}+\boldsymbol{\mu}-\left(m_{1} / M_{2}\right) \boldsymbol{\lambda}\right|}\right) .
$$

Let $\mathbf{M}=\mathbb{R}^{15}$, and we consider the manifold $(\mathbf{M},\{\cdot, \cdot\}, \mathscr{H})$, with Poisson brackets $\{\cdot, \cdot\}$ defined by means of the Poisson tensor

$$
\mathbf{B}(\mathbf{z})=\left(\begin{array}{ccccc}
\hat{\boldsymbol{\Pi}} & \hat{\lambda} & \widehat{\mathbf{p}_{\lambda}} & \hat{\boldsymbol{\mu}} & \widehat{\mathbf{p}_{\mu}} \\
\hat{\lambda} & \mathbf{0} & \mathbf{I}_{\mathbb{R}^{3}} & \mathbf{0} & \mathbf{0} \\
\widehat{\mathbf{p} \lambda} & -\mathbf{I}_{\mathbb{R}^{3}} & \mathbf{0} & \mathbf{0} & \mathbf{0} \\
\widehat{\boldsymbol{\mu}} & \mathbf{0} & \mathbf{0} & \mathbf{0} & \mathbf{I}_{\mathbb{R}^{3}} \\
\widehat{\mathbf{p}_{\mu}} & \mathbf{0} & \mathbf{0} & -\mathbf{I}_{\mathbb{R}^{3}} & \mathbf{0}
\end{array}\right)
$$


4 Eulerian equilibria for a gyrostat in the three-body problem

In $\mathbf{B}(\mathbf{z}), \hat{\mathbf{v}}$ is considered to be the image of the vector $\mathbf{v} \in \mathbb{R}^{3}$ by the standard isomorphism between the Lie Algebras $\mathbb{R}^{3}$ and $\mathfrak{s o}(3)$, that is,

$$
\widehat{\mathbf{v}}=\left(\begin{array}{ccc}
0 & -v_{3} & v_{2} \\
v_{3} & 0 & -v_{1} \\
-v_{2} & v_{1} & 0
\end{array}\right)
$$

The equations of the motion are given by the following expression:

$$
\frac{d \mathbf{z}}{d t}=\{\mathbf{z}, \mathscr{H}(\mathbf{z})\} \quad(\mathbf{z})=\mathbf{B}(\mathbf{z}) \nabla_{\mathbf{z}} \mathscr{H}(\mathbf{z})
$$

where $\nabla_{\mathbf{z}} f$ is the gradient of $f \in C^{\infty}(\mathbf{M})$ with respect to an arbitrary vector $\mathbf{z}$.

Developing $\{\mathbf{z}, \mathcal{H}(\mathbf{z})\}$, we obtain the following group of vectorial equations of the motion:

$$
\begin{gathered}
\frac{d \boldsymbol{\Pi}}{d t}=\boldsymbol{\Pi} \times \boldsymbol{\Omega}+\boldsymbol{\lambda} \times \nabla_{\lambda} \mathscr{V}+\boldsymbol{\mu} \times \nabla_{\boldsymbol{\mu}} \mathscr{V}, \\
\frac{d \boldsymbol{\lambda}}{d t}=\frac{\mathbf{p}_{\lambda}}{g_{1}}+\lambda \times \boldsymbol{\Omega}, \quad \frac{d \mathbf{p}_{\lambda}}{d t}=\mathbf{p}_{\lambda} \times \mathbf{\Omega}-\nabla_{\lambda} \mathscr{V}, \\
\frac{d \boldsymbol{\mu}}{d t}=\frac{\mathbf{p}_{\mu}}{g_{2}}+\boldsymbol{\mu} \times \mathbf{\Omega}, \quad \frac{d \mathbf{p}_{\boldsymbol{\mu}}}{d t}=\mathbf{p}_{\boldsymbol{\mu}} \times \mathbf{\Omega}-\nabla_{\boldsymbol{\mu}} \mathscr{V} .
\end{gathered}
$$

Important elements of $\mathbf{B}(\mathbf{z})$ are the associate Casimir functions. We consider the total angular momentum $\mathbf{L}$ given by

$$
\mathrm{L}=\Pi+\lambda \times \mathbf{p}_{\lambda}+\mu \times \mathbf{p}_{\mu}
$$

Then the following result is verified (see Vera and Vigueras [6] for details).

Proposition 2.1. If $\varphi$ is a real smooth function not constant, then $\varphi\left(|\mathbf{L}|^{2} / 2\right)$ is a Casimir function of the Poisson tensor $\mathbf{B}(\mathbf{z})$. Moreover $\operatorname{Ker} \mathbf{B}(\mathbf{z})=\left\langle\nabla_{\mathbf{z}} \varphi\right\rangle$. Also, $d \mathbf{L} / d t=\mathbf{0}$, that is to say the total angular momentum vector remains constant.

2.1. Approximate Poisson dynamics. To simplify the problem we assume that the gyrostat $S_{0}$ is symmetrical around the third axis of inertia $O Z$ and with respect to the plane $O X Y$ being $O X, O Y, O Z$ are the coordinated axes of the body frame $\mathfrak{J}$. If the mutual distances are bigger than the individual dimensions of the bodies, then we can develop the potential in fast convergent series. Under these hypotheses, we will be able to carry out a study of equilibria in different approximate dynamics.

Applying the Legendre development of the potential, we have

$$
\mathscr{V}(\boldsymbol{\lambda}, \boldsymbol{\mu})=-\left(\frac{G m_{1} m_{2}}{|\boldsymbol{\lambda}|}+\sum_{i=0}^{\infty} \frac{G m_{1} A_{2 i}}{\left|\boldsymbol{\mu}+\left(m_{2} / M_{2}\right) \boldsymbol{\lambda}\right|^{2 i+1}}+\sum_{i=0}^{\infty} \frac{G m_{2} A_{2 i}}{\left|\boldsymbol{\mu}-\left(m_{1} / M_{2}\right) \boldsymbol{\lambda}\right|^{2 i+1}}\right),
$$

where $A_{0}=m_{0}, A_{2}=(C-A) / 2$ and $A_{2 i}$ are certain coefficients related to the geometry of the gyrostat, see Vera and Vigueras [6] for details. 
Definition 2.2. We call approximate potential of order $k$ to the following expression:

$$
\mathscr{V}_{k}(\boldsymbol{\lambda}, \boldsymbol{\mu})=-\left(\frac{G m_{1} m_{2}}{|\boldsymbol{\lambda}|}+\sum_{i=0}^{k} \frac{G m_{1} A_{2 i}}{\left|\boldsymbol{\mu}+\left(m_{2} / M_{2}\right) \boldsymbol{\lambda}\right|^{2 i+1}}+\sum_{i=0}^{k} \frac{G m_{2} A_{2 i}}{\left|\boldsymbol{\mu}-\left(m_{1} / M_{2}\right) \boldsymbol{\lambda}\right|^{2 i+1}}\right)
$$

It is easy to demonstrate the following lemmas.

LEMMA 2.3. Given the approximate potential of order $k$,

$$
\begin{aligned}
\nabla_{\lambda} \mathscr{V}_{k}= & \frac{G m_{1} m_{2} \lambda}{|\lambda|^{3}}+\frac{G m_{1} m_{2}}{M_{2}} \sum_{i=0}^{k} \frac{\left(\boldsymbol{\mu}+\left(m_{2} / M_{2}\right) \lambda\right)(2 i+1) A_{2 i}}{\left|\boldsymbol{\mu}+\left(m_{2} / M_{2}\right) \lambda\right|^{2 i+3}} \\
& -\frac{G m_{1} m_{2}}{M_{2}} \sum_{i=0}^{k} \frac{\left(\boldsymbol{\mu}-\left(m_{1} / M_{2}\right) \boldsymbol{\lambda}\right)(2 i+1) A_{2 i}}{\left|\boldsymbol{\mu}-\left(m_{1} / M_{2}\right) \boldsymbol{\lambda}\right|^{2 i+3}} \\
\nabla_{\mu} \mathscr{V}_{k}= & G m_{1} \sum_{i=0}^{k} \frac{\left(\boldsymbol{\mu}+\left(m_{2} / M_{2}\right) \lambda\right)(2 i+1) A_{2 i}}{\left|\boldsymbol{\mu}+\left(m_{2} / M_{2}\right) \boldsymbol{\lambda}\right|^{2 i+3}}+G m_{2} \sum_{i=0}^{k} \frac{\left(\boldsymbol{\mu}-\left(m_{1} / M_{2}\right) \lambda\right)(2 i+1) A_{2 i}}{\left|\boldsymbol{\mu}-\left(m_{1} / M_{2}\right) \boldsymbol{\lambda}\right|^{2 i+3}} .
\end{aligned}
$$

The following identities are verified

$$
\nabla_{\lambda} \mathscr{V}_{k}=\widetilde{A}_{11} \lambda+\widetilde{A}_{12} \boldsymbol{\mu}, \quad \nabla_{\mu} \mathscr{V}_{k}=\widetilde{A}_{21} \lambda+\widetilde{A}_{22} \boldsymbol{\mu}
$$

being

$$
\begin{aligned}
\tilde{A}_{11}(\boldsymbol{\lambda}, \boldsymbol{\mu})= & \frac{G m_{1} m_{2}}{|\boldsymbol{\lambda}|^{3}}+\frac{G m_{1} m_{2}^{2}}{M_{2}^{2}}\left(\sum_{i=0}^{k} \frac{\beta_{i}}{\left|\boldsymbol{\mu}+\left(m_{2} / M_{2}\right) \boldsymbol{\lambda}\right|^{2 i+3}}\right) \\
& +\frac{G m_{1}^{2} m_{2}}{M_{2}^{2}}\left(\sum_{i=0}^{k} \frac{\beta_{i}}{\left|\boldsymbol{\mu}-\left(m_{1} / M_{2}\right) \boldsymbol{\lambda}\right|^{2 i+3}}\right), \\
\tilde{A}_{12}(\boldsymbol{\lambda}, \boldsymbol{\mu})= & \frac{G m_{1} m_{2}}{M_{2}}\left(\sum_{i=0}^{k} \frac{\beta_{i}}{\left|\boldsymbol{\mu}+\left(m_{2} / M_{2}\right) \boldsymbol{\lambda}\right|^{2 i+3}}-\sum_{i=0}^{k} \frac{\beta_{i}}{\left|\boldsymbol{\mu}-\left(m_{1} / M_{2}\right) \boldsymbol{\lambda}\right|^{2 i+3}}\right), \\
\tilde{A}_{22}(\boldsymbol{\lambda}, \boldsymbol{\mu})= & G m_{1}\left(\sum_{i=0}^{k} \frac{\beta_{i}}{\left|\boldsymbol{\mu}+\left(m_{2} / M_{2}\right) \boldsymbol{\lambda}\right|^{2 i+3}}\right)+G m_{2}\left(\sum_{i=0}^{k} \frac{\beta_{i}}{\left|\boldsymbol{\mu}-\left(m_{1} / M_{2}\right) \boldsymbol{\lambda}\right|^{2 i+3}}\right), \\
\tilde{A}_{21}(\boldsymbol{\lambda}, \boldsymbol{\mu})= & \widetilde{A}_{12}(\boldsymbol{\lambda}, \boldsymbol{\mu})
\end{aligned}
$$

with coefficients $\beta_{0}=m_{0}, \beta_{1}=3 / 2(C-A), \beta_{i}=(2 i+1) A_{2 i}$ for $i \geqslant 1$. 
6 Eulerian equilibria for a gyrostat in the three-body problem

Definition 2.4. Let $\mathbf{M}=\mathbb{R}^{15}$ and the manifold $\left(\mathbf{M},\{\cdot, \cdot\}, \mathscr{H}_{k}\right)$, with Poisson brackets $\{\cdot, \cdot\}$, defined by means of the Poisson tensor (2.4). We call approximate dynamics of order $k$ to the differential equations of motion given by the following expression:

$$
\frac{d \mathbf{z}}{d t}=\left\{\mathbf{z}, \mathscr{H}_{k}(\mathbf{z})\right\}, \quad(\mathbf{z})=\mathbf{B}(\mathbf{z}) \nabla_{\mathbf{z}} \mathscr{H}_{k}(\mathbf{z})
$$

being

$$
\mathscr{H}_{k}(\mathbf{z})=\frac{\left|\mathbf{p}_{\boldsymbol{\lambda}}\right|^{2}}{2 g_{1}}+\frac{\left|\mathbf{p}_{\boldsymbol{\mu}}\right|^{2}}{2 g_{2}}+\frac{1}{2} \Pi \square^{-1} \Pi-\mathbf{l}_{r} \cdot \square^{-1} \Pi+\mathscr{V}_{k}(\boldsymbol{\lambda}, \boldsymbol{\mu}) .
$$

2.1.1. Integrals of the system. On the other hand, it is easy to verify that

$$
\nabla_{\mathbf{z}}\left(|\boldsymbol{\Pi}|^{2}\right) \mathbf{B}(\mathbf{z}) \nabla_{\mathbf{z}} \mathscr{H}_{0}(\mathbf{z})=0
$$

and similarly when the gyrostat is of revolution

$$
\nabla_{\mathbf{z}}\left(\Pi_{3}\right) \mathbf{B}(\mathbf{z}) \nabla_{\mathbf{z}} \mathscr{H}_{k}(\mathbf{z})=0
$$

where $\pi_{3}$ is the third component of the rotational angular momentum of the gyrostat. It is verified the following result.

THeOREM 2.5. In the approximate dynamics of order $0,|\Pi|^{2}$ is an integral of motion and also when the gyrostat is of revolution $\pi_{3}$ is another integral of motion.

2.2. Relative equilibria. The relative equilibria are the equilibria of the twice reduced problem whose Hamiltonian function is obtained in Vera and Vigueras [6] for the case $n=2$. If we denote by $\mathbf{z}_{e}=\left(\boldsymbol{\Pi}_{e}, \lambda^{e}, \mathbf{p}_{\lambda}^{e}, \boldsymbol{\mu}^{e}, \mathbf{p}_{\mu}^{e}\right)$ a generic relative equilibrium of an approximate dynamics of order $k$, then this verifies the equations

$$
\begin{array}{cc}
\boldsymbol{\Pi}_{e} \times \boldsymbol{\Omega}_{e}+\lambda^{e} \times\left(\nabla_{\lambda} \mathscr{V}_{k}\right)_{e}+\boldsymbol{\mu}^{e} \times\left(\nabla_{\boldsymbol{\mu}} \mathscr{V}_{k}\right)_{e}=\mathbf{0}, \\
\frac{\mathbf{p}_{\lambda}^{e}}{g_{1}}+\lambda^{e} \times \boldsymbol{\Omega}_{e}=\mathbf{0}, \quad \mathbf{p}_{\lambda}^{e} \times \boldsymbol{\Omega}_{e}=\left(\nabla_{\lambda} \mathscr{V}_{k}\right)_{e} \\
\frac{\mathbf{p}_{\boldsymbol{\mu}}^{e}}{g_{2}}+\boldsymbol{\mu}^{e} \times \boldsymbol{\Omega}_{e}=\mathbf{0}, & \mathbf{p}_{\boldsymbol{\mu}}^{e} \times \boldsymbol{\Omega}_{e}=\left(\nabla_{\boldsymbol{\mu}} \mathscr{V}_{k}\right)_{e}
\end{array}
$$

Also by virtue of the relationships obtained in Vera and Vigueras [6], we have the following result.

Lemma 2.6. If $\mathbf{z}_{e}=\left(\boldsymbol{\Pi}_{e}, \lambda^{e}, \mathbf{p}_{\lambda}^{e}, \boldsymbol{\mu}^{e}, \mathbf{p}_{\mu}^{e}\right)$ is a relative equilibrium of an approximate dynamics of order $k$, the following relationships are verified:

$$
\begin{aligned}
& \left|\boldsymbol{\Omega}_{e}\right|^{2}\left|\lambda^{e}\right|^{2}-\left(\lambda^{e} \cdot \boldsymbol{\Omega}_{e}\right)^{2}=\frac{1}{g_{1}}\left(\lambda^{e} \cdot\left(\nabla_{\lambda} \mathscr{V}_{k}\right)_{e}\right), \\
& \left|\boldsymbol{\Omega}_{e}\right|^{2}\left|\boldsymbol{\mu}^{e}\right|^{2}-\left(\boldsymbol{\mu}^{e} \cdot \boldsymbol{\Omega}_{e}\right)^{2}=\frac{1}{g_{2}}\left(\boldsymbol{\mu}^{e} \cdot\left(\nabla_{\boldsymbol{\mu}} \mathscr{V}_{k}\right)_{e}\right) .
\end{aligned}
$$


The last two previous identities will be used to obtain necessary conditions for the existence of relative equilibria in this approximate dynamics.

We will study certain relative equilibria in the approximate dynamics supposing that the vectors $\boldsymbol{\Omega}_{e}, \lambda^{e}, \boldsymbol{\mu}^{e}$ satisfy special geometric properties.

Definition 2.7. $\mathbf{z}_{e}$ is an Eulerian relative equilibrium in an approximate dynamics of order $k$ when $\lambda^{e}$ and $\boldsymbol{\mu}^{e}$ are proportional and $\boldsymbol{\Omega}_{e}$ is perpendicular to the straight line that they generate.

Remark 2.8. The previous hypotheses simplify the conditions of Lemma 2.6. In a next paper we will study the possible "inclined" relative equilibria, in which $\boldsymbol{\Omega}_{e}$ form an angle $\alpha \neq 0$ and $\pi / 2$ with the vector $\lambda^{e}$.

From the equations of motion, the following property is deduced.

Proposition 2.9. In a Eulerian relative equilibrium for any approximate dynamics of arbitrary order, moments are not exercised on the gyrostat.

Next we obtain necessary and sufficient conditions for the existence of Eulerian relative equilibria.

\section{Relative equilibria of Euler type}

According to the relative position of the gyrostat $S_{0}$ with respect to $S_{1}$ and $S_{2}$, there are three possible equilibrium configurations (see Figure 3.1): (a) $S_{0} S_{2} S_{1}$, (b) $S_{2} S_{0} S_{1}$, and (c) $S_{2} S_{1} S_{0}$.

\subsection{Necessary condition of existence}

Lemma 3.1. If $\mathbf{z}_{e}=\left(\boldsymbol{\Pi}_{e}, \lambda^{e}, \mathbf{p}_{\lambda}^{e}, \boldsymbol{\mu}^{e}, \mathbf{p}_{\mu}^{e}\right)$ is a relative equilibrium of Euler type, then for the configuration $S_{0} S_{2} S_{1}$,

$$
\left|\boldsymbol{\mu}^{e}+\frac{m_{1}}{M_{2}} \lambda^{e}\right|=\left|\boldsymbol{\lambda}^{e}\right|+\left|\boldsymbol{\mu}^{e}-\frac{m_{2}}{M_{2}} \boldsymbol{\lambda}^{e}\right| .
$$

In a similar way, for the configuration $S_{2} S_{0} S_{1}$,

$$
\left|\lambda^{e}\right|=\left|\boldsymbol{\mu}^{e}-\frac{m_{1}}{M_{2}} \lambda^{e}\right|+\left|\boldsymbol{\mu}^{e}+\frac{m_{2}}{M_{2}} \lambda^{e}\right|
$$

Finally, for the configuration $S_{2} S_{1} S_{0}$,

$$
\left|\boldsymbol{\mu}^{e}-\frac{m_{2}}{M_{2}} \lambda^{e}\right|=\left|\boldsymbol{\mu}^{e}+\frac{m_{1}}{M_{2}} \lambda^{e}\right|+\left|\lambda^{e}\right|
$$

Next we study necessary and sufficient conditions for the existence of relative equilibria of Euler type for the configuration $S_{0} S_{2} S_{1}$; the other configurations are studied in a similar way. If $\mathbf{z}_{e}$ is a relative equilibrium of Euler type, in the configuration $S_{0} S_{2} S_{1}$ in an 
8 Eulerian equilibria for a gyrostat in the three-body problem

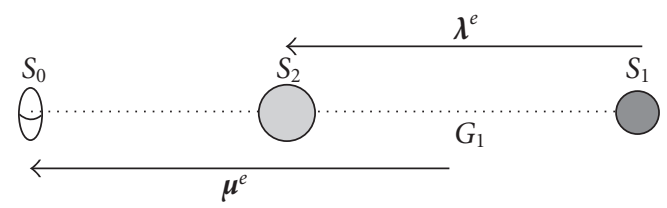

(a)

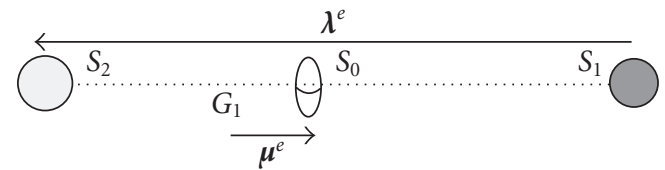

(b)

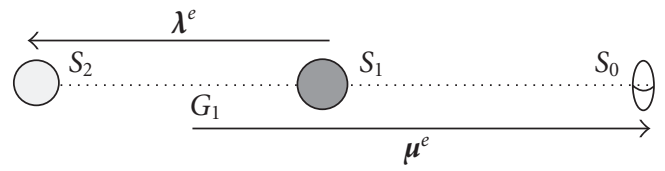

(c)

Figure 3.1. Eulerian configurations.

approximate dynamics of order $k$, we have

$$
\begin{gathered}
g_{1}\left|\boldsymbol{\Omega}_{e}\right|^{2}\left|\lambda^{e}\right|^{2}=\lambda^{e} \cdot\left(\nabla_{\lambda} \mathscr{V}_{k}\right)_{e}, \quad g_{2}\left|\boldsymbol{\Omega}_{e}\right|^{2}\left|\boldsymbol{\mu}^{e}\right|^{2}=\boldsymbol{\mu}^{e} \cdot\left(\nabla_{\boldsymbol{\mu}} \mathscr{V}_{k}\right)_{e}, \\
\boldsymbol{\mu}^{e}-\frac{m_{1}}{M_{2}} \lambda^{e}=\rho \lambda^{e}, \quad \boldsymbol{\mu}^{e}+\frac{m_{2}}{M_{2}} \lambda^{e}=(1+\rho) \lambda^{e}, \quad \boldsymbol{\mu}^{e}=\frac{\left((1+\rho) m_{1}+\rho m_{2}\right)}{M_{2}} \lambda^{e},
\end{gathered}
$$

where $\rho \in(0,+\infty)$ in the case (a), $\rho \in(-1,0)$ in the case (b), and $\rho \in(-\infty,-1)$ in the case (c). And it is possible to obtain the following expressions:

$$
\left(\nabla_{\lambda} \mathscr{V}_{k}\right)_{e}=f_{1}(\rho) \lambda^{e}, \quad\left(\nabla_{\mu} \mathscr{V}_{k}\right)_{e}=f_{2}(\rho) \lambda^{e}
$$

where

$$
\begin{aligned}
& f_{1}(\rho)=\frac{G m_{1} m_{2}}{\left|\lambda^{e}\right|^{3}}+\frac{G m_{1} m_{2}}{M_{2}}\left(\sum_{i=0}^{k} \frac{\beta_{i}}{\left|\lambda^{e}\right|^{2 i+3}}\left(\frac{1+\rho}{|1+\rho|^{2 i+3}}-\frac{\rho}{|\rho|^{2 i+3}}\right)\right), \\
& f_{2}(\rho)=\sum_{i=0}^{k} \frac{G \beta_{i}}{\left|\lambda^{e}\right|^{2 i+3}}\left(\frac{m_{1}(1+\rho)}{|1+\rho|^{2 i+3}}+\frac{m_{2} \rho}{|\rho|^{2 i+3}}\right) .
\end{aligned}
$$


Restricting us to the case (a) we have

$$
\begin{aligned}
& f_{1}(\rho)=\frac{G m_{1} m_{2}}{\left|\lambda^{e}\right|^{3}}+\frac{G m_{1} m_{2}}{M_{2}}\left(\sum_{i=0}^{k} \frac{\beta_{i}}{\left|\lambda^{e}\right|^{2 i+3}}\left(\frac{1}{(1+\rho)^{2 i+2}}-\frac{1}{\rho^{2 i+2}}\right)\right), \\
& f_{2}(\rho)=\sum_{i=0}^{k} \frac{G \beta_{i}}{\left|\lambda^{e}\right|^{2 i+3}}\left(\frac{m_{1}}{(1+\rho)^{2 i+2}}+\frac{m_{2}}{\rho^{2 i+2}}\right) .
\end{aligned}
$$

Now, from the identities

$$
\lambda^{e} \cdot\left(\nabla_{\lambda} \mathscr{V}_{k}\right)_{e}=\left|\lambda^{e}\right|^{2} f_{1}(\rho), \quad \boldsymbol{\mu}^{e} \cdot\left(\nabla_{\mu} \mathscr{V}_{k}\right)_{e}=\frac{\left((1+\rho) m_{1}+\rho m_{2}\right)}{M_{2}}\left|\lambda^{e}\right|^{2} f_{2}(\rho)
$$

we deduce the following equations:

$$
\left|\boldsymbol{\Omega}_{e}\right|^{2}=\frac{f_{1}(\rho)}{g_{1}}, \quad\left|\boldsymbol{\Omega}_{e}\right|^{2}=\frac{M_{2} f_{2}(\rho)}{g_{2}\left((1+\rho) m_{1}+\rho m_{2}\right)} .
$$

Then for a relative equilibrium of Euler type $\rho$ must be a positive real root of the following equation:

$$
m_{0}\left(m_{1}+m_{2}\right)\left((1+\rho) m_{1}+\rho m_{2}\right) f_{1}(\rho)=m_{1} m_{2}\left(m_{0}+m_{1}+m_{2}\right) f_{2}(\rho) .
$$

We summarize all these results in the following proposition.

Proposition 3.2. If $\mathbf{z}_{e}=\left(\boldsymbol{\Pi}_{e}, \boldsymbol{\lambda}^{e}, \mathbf{p}_{\lambda}^{e}, \boldsymbol{\mu}^{e}, \mathbf{p}_{\mu}^{e}\right)$ is an Eulerian relative equilibrium in the configuration $S_{0} S_{2} S_{1}$, (3.10) has, at least, a positive real root; where the functions $f_{1}(\rho)$ and $f_{2}(\rho)$ are given by (3.7) and the modulus of the angular velocity of the gyrostat is

$$
\left|\boldsymbol{\Omega}_{e}\right|^{2}=\frac{f_{1}(\rho)}{g_{1}} .
$$

Remark 3.3. If a solution of relative equilibrium of Euler type exists, in an approximate dynamics of order $k$, fixing $\left|\boldsymbol{\lambda}_{e}\right|$, (3.10) has positive real solutions. The number of real roots of (3.10) will depend, obviously, on the numerous parameters that exist in our system. Similar results would be obtained for the other two cases.

3.2. Sufficient condition of existence. The following proposition indicates how to find solutions of (2.18).

Proposition 3.4. Fixing $\left|\lambda^{e}\right|$, let $\rho$ be a solution of (3.10) where the functions $f_{1}(\rho)$ and $f_{2}(\rho)$ are given for the case (a) as (3.7) then $\mathbf{z}_{e}=\left(\boldsymbol{\Pi}_{e}, \lambda^{e}, \mathbf{p}_{\lambda}^{e}, \boldsymbol{\mu}^{e}, \mathbf{p}_{\mu}^{e}\right)$, given by

$$
\begin{aligned}
& \lambda^{e}=\left(\lambda^{e}, 0,0\right), \quad \boldsymbol{\mu}^{e}=\left(\mu^{e}, 0,0\right), \quad \boldsymbol{\Omega}_{e}=\left(0,0, \omega_{e}\right), \\
& \mathbf{p}_{\lambda}^{e}=\left(0, g_{1} \omega_{e} \lambda^{e}, 0\right), \quad \mathbf{p}_{\mu}^{e}=\left(0, g_{2} \omega_{e} \mu^{e}, 0\right), \quad \Pi_{e}=\left(0,0, C \omega_{e}+l\right),
\end{aligned}
$$


10 Eulerian equilibria for a gyrostat in the three-body problem

where

$$
\boldsymbol{\mu}^{e}=\frac{\left((1+\rho) m_{1}+\rho m_{2}\right)}{M_{2}} \lambda^{e}, \quad \omega_{e}^{2}=\frac{f_{1}(\rho)}{g_{1}},
$$

is a solution of relative equilibrium of Euler type, in an approximate dynamics of order $k$ in the configuration $S_{0} S_{2} S_{1}$. The total angular momentum of the system is given by

$$
\mathbf{L}=\left(0,0, C \omega_{e}+l+g_{1} \omega_{e} \lambda^{e}+g_{2} \omega_{e} \mu^{e}\right),
$$

where $l$ is the gyrostatic momentum.

Let us see the existence and number of solutions for the approximate dynamics of orders zero and one, respectively. For superior order it is possible to use a similar technical.

\section{Eulerian equilibria in an approximate dynamics of orders zero and one}

For the configuration $S_{0} S_{2} S_{1}$, in an approximate dynamics of order zero, we have

$$
\begin{aligned}
& f_{1}(\rho)=\frac{G m_{1} m_{2}}{\left|\lambda_{e}\right|^{3}}\left(1+\frac{m_{0}}{M_{2}}\left(\frac{1}{(1+\rho)^{2}}-\frac{1}{\rho^{2}}\right)\right), \\
& f_{2}(\rho)=\frac{G m_{0}}{\left|\lambda_{e}\right|^{3}}\left(\frac{m_{1}}{(1+\rho)^{2}}+\frac{m_{2}}{\rho^{2}}\right) .
\end{aligned}
$$

Equation (3.10) is equivalent to the following polynomial equation:

$$
\begin{aligned}
\left(m_{1}+m_{2}\right) \rho^{5}+\left(3 m_{1}+2 m_{2}\right) \rho^{4}+\left(3 m_{1}+m_{2}\right) \rho^{3} & \\
& -\left(3 m_{0}+m_{2}\right) \rho^{2}-\left(3 m_{0}+2 m_{2}\right) \rho-\left(m_{0}+m_{2}\right)=0 .
\end{aligned}
$$

This equation has an unique positive real solution, then in this case for the approximate dynamics of order zero, there exists a unique relative equilibrium of Euler type.

On the other hand, one has

$$
\left|\boldsymbol{\Omega}_{e}\right|^{2}=\frac{G\left(m_{1}+m_{2}\right)}{\left|\lambda^{e}\right|^{3}}\left(1+\frac{m_{0}}{m_{1}+m_{2}}\left(\frac{1}{(1+\rho)^{2}}-\frac{1}{\rho^{2}}\right)\right),
$$

$\rho$ being the only one positive solution of (4.2).

The following proposition gathers the results about relative equilibria of Euler type in an approximate dynamics of order zero in any of the previously mentioned cases (a), (b), or $(c)$.

Proposition 4.1. (1) If $\rho$ is the unique positive root of (4.2) with $\left|\Omega_{e}\right|^{2}$ being expressed as (4.3), then $\mathbf{z}_{e}=\left(\boldsymbol{\Pi}_{e}, \lambda^{e}, \mathbf{p}_{\lambda}^{e}, \boldsymbol{\mu}^{e}, \mathbf{p}_{\mu}^{e}\right)$, given by

$$
\begin{gathered}
\lambda^{e}=\left(\lambda^{e}, 0,0\right), \quad \boldsymbol{\mu}^{e}=\left(\mu^{e}, 0,0\right), \quad \boldsymbol{\Omega}_{e}=\left(0,0, \omega_{e}\right), \\
\mathbf{p}_{\lambda}^{e}=\left(0, g_{1} \omega_{e} \lambda^{e}, 0\right), \quad \mathbf{p}_{\boldsymbol{\mu}}^{e}=\left(0, g_{2} \omega_{e} \mu^{e}, 0\right), \quad \boldsymbol{\Pi}_{e}=\left(0,0, C \omega_{e}+l\right),
\end{gathered}
$$

is the unique solution of relative equilibrium of Euler type in the configuration $S_{0} S_{2} S_{1}$. 
(2) If $\rho \in(-1,0)$ is the unique root of the equation

$$
\begin{aligned}
& \left(m_{1}+m_{2}\right) \rho^{5}+\left(3 m_{1}+2 m_{2}\right) \rho^{4}+\left(3 m_{1}+m_{2}\right) \rho^{3} \\
& \quad+\left(3 m_{0}+2 m_{1}+m_{2}\right) \rho^{2}+\left(3 m_{0}+2 m_{2}\right) \rho+\left(m_{0}+m_{2}\right)=0
\end{aligned}
$$

with

$$
\left|\boldsymbol{\Omega}_{e}\right|^{2}=\frac{G\left(m_{1}+m_{2}\right)}{\left|\boldsymbol{\lambda}_{e}\right|^{3}}\left(1+\frac{m_{0}}{m_{1}+m_{2}}\left(\frac{1}{\rho^{2}}-\frac{1}{(1+\rho)^{2}}\right)\right),
$$

then $\mathbf{z}_{e}=\left(\boldsymbol{\Pi}_{e}, \lambda^{e}, \mathbf{p}_{\lambda}^{e}, \boldsymbol{\mu}^{e}, \mathbf{p}_{\mu}^{e}\right)$, given by

$$
\begin{aligned}
& \lambda^{e}=\left(\lambda^{e}, 0,0\right), \quad \boldsymbol{\mu}^{e}=\left(\mu^{e}, 0,0\right), \quad \boldsymbol{\Omega}_{e}=\left(0,0, \omega_{e}\right), \\
& \mathbf{p}_{\lambda}^{e}=\left(0, g_{1} \omega_{e} \lambda^{e}, 0\right), \quad \mathbf{p}_{\mu}^{e}=\left(0, g_{2} \omega_{e} \mu^{e}, 0\right), \quad \Pi_{e}=\left(0,0, C \omega_{e}+l\right),
\end{aligned}
$$

is the unique solution of relative equilibrium of Euler type in the configuration $S_{2} S_{0} S_{1}$.

(3) If $\rho \in(-\infty,-1)$ is the unique root of the equation

$$
\begin{aligned}
\left(m_{1}+m_{2}\right) \rho^{5}+\left(3 m_{1}+2 m_{2}\right) \rho^{4}+\left(2 m_{0}+3 m_{1}+m_{2}\right) \rho^{3} \\
+\left(3 m_{0}+m_{2}\right) \rho^{2}+\left(3 m_{0}+2 m_{2}\right) \rho+\left(m_{0}+m_{2}\right)=0
\end{aligned}
$$

with

$$
\left|\boldsymbol{\Omega}_{e}\right|^{2}=\frac{G\left(m_{1}+m_{2}\right)}{\left|\boldsymbol{\lambda}_{e}\right|^{3}}\left(1+\frac{m_{0}}{m_{1}+m_{2}}\left(\frac{1}{\rho^{2}}+\frac{1}{(1+\rho)^{2}}\right)\right),
$$

then $\mathbf{z}_{e}=\left(\boldsymbol{\Pi}_{e}, \lambda^{e}, \mathbf{p}_{\lambda}^{e}, \boldsymbol{\mu}^{e}, \mathbf{p}_{\mu}^{e}\right)$, given by

$$
\begin{gathered}
\lambda^{e}=\left(\lambda^{e}, 0,0\right), \quad \boldsymbol{\mu}^{e}=\left(\mu^{e}, 0,0\right), \quad \boldsymbol{\Omega}_{e}=\left(0,0, \omega_{e}\right), \\
\mathbf{p}_{\lambda}^{e}=\left(0, g_{1} \omega_{e} \lambda^{e}, 0\right), \quad \mathbf{p}_{\mu}^{e}=\left(0, g_{2} \omega_{e} \mu^{e}, 0\right), \quad \Pi_{e}=\left(0,0, C \omega_{e}+l\right),
\end{gathered}
$$

is the unique solution of relative equilibrium of Euler type in the configuration $S_{2} S_{1} S_{0}$.

Remark 4.2. If $m_{0} \rightarrow 0$, then $\left|\Omega_{e}\right|^{2}=G\left(m_{1}+m_{2}\right) /\left|\lambda_{e}\right|^{3}$ and the equations that determine the Eulerian equilibria are the same ones of the restricted three-body problem.

4.1. Number of Eulerian equilibria in an approximate dynamics of order one. For the approximate dynamics of order one, after carrying out the appropriate calculations, (3.10) corresponding to the configuration $S_{0} S_{2} S_{1}$ is reduced to the study of the positive 
12 Eulerian equilibria for a gyrostat in the three-body problem

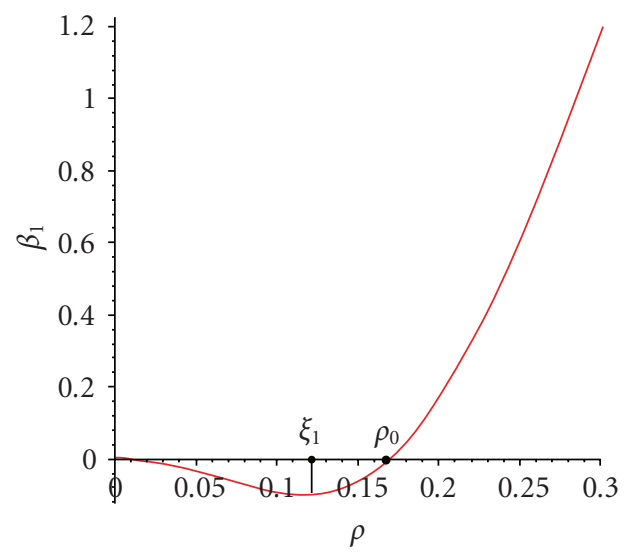

Figure 4.1. Function $R_{1}(\rho)$.

real roots of the polynomial

$$
\begin{aligned}
p_{1}(\rho)= & m_{0} a^{2}\left(m_{1}+m_{2}\right) \rho^{9}+m_{0} a^{2}\left(5 m_{1}+4 m_{2}\right) \rho^{8}+m_{0} a^{2}\left(10 m_{1}+6 m_{2}\right) \rho^{7} \\
& +3 m_{0} a^{2}\left(3 m_{1}+m_{2}-m_{0}\right) \rho^{6}+3 m_{0} a^{2}\left(m_{1}-m_{2}-3 m_{0}\right) \rho^{5} \\
& -\left(6 m_{0} m_{2} a^{2}+10 m_{0}^{2} a^{2}+\beta_{1}\left(m_{1}+m_{2}+5 m_{0}\right)\right) \rho^{4} \\
& -\left(4 m_{0} m_{2} a^{2}+5 m_{0}^{2} a^{2}+\beta_{1}\left(10 m_{0}+4 m_{2}\right)\right) \rho^{3} \\
& -\left(m_{0} m_{2} a^{2}+m_{0}^{2} a^{2}+\beta_{1}\left(6 m_{2}+10 m_{0}\right)\right) \rho^{2} \\
& -\beta_{1}\left(5 m_{0}+4 m_{2}\right) \rho-\beta_{1}\left(m_{0}+m_{2}\right),
\end{aligned}
$$

where $a=\left|\lambda_{e}\right|$ and $\beta_{1}=3(C-A) / 2, C$ and $A$ being the principal moments of inertia of the gyrostat.

To study the positive real roots of this equation, after a detailed analysis of the same one, it can be expressed in the following way:

$$
\beta_{1}=R_{1}(\rho)=\frac{m_{0} a^{2} \rho^{2}(\rho+1)^{2} p_{0}(\rho)}{q_{0}(\rho)}
$$

Where $\beta_{1}=3(C-A) / 2, p_{0}$ is the polynomial of grade five that determines the relative equilibria in the approximate dynamics of order 0 , that is given by formula (4.2), and the polynomial $q_{0}$ comes determined by the following expression:

$$
\begin{aligned}
q_{0}(\rho)= & \left(m_{1}+m_{2}+5 m_{0}\right) \rho^{4}+\left(4 m_{2}+10 m_{0}\right) \rho^{3} \\
& +\left(6 m_{2}+10 m_{0}\right) \rho^{2}+\left(4 m_{2}+5 m_{0}\right) \rho+\left(m_{0}+m_{2}\right) .
\end{aligned}
$$

The rational function $R_{1}(\rho)$, for any value of $m_{0}, m_{1}, m_{2}$, always presents a minimum $\xi_{1}$ located among 0 and $\rho_{0}$, with this last value being the only one positive zero of the polynomial $p_{0}(\rho)$.

By virtue of these statements, the following result is obtained (see Figure 4.1). 
Proposition 4.3. In the approximate dynamics of order one, if the gyrostat $S_{0}$ is prolate $\left(\beta_{1}<0\right)$, the following hold:

(1) $\beta_{1}<R_{1}\left(\xi_{1}\right)$, then relative equilibria of Euler type do not exist.

(2) $\beta_{1}=R_{1}\left(\xi_{1}\right)$, then there exists an only relative equilibrium of Euler type.

(3) $R_{1}\left(\xi_{1}\right)<\beta_{1}<0$, then two 1-parametric families of relative equilibria of Euler type exist.

If $S_{0}$ is oblate $\left(\beta_{1}>0\right)$, then there exists a unique 1-parametric family of relative equilibria of Euler type.

Similarly for the configuration $S_{2} S_{0} S_{1}$ we obtain the following result (see Figures A.1 and A.2).

Proposition 4.4. In the approximate dynamics of order one, if $m_{1} \neq m_{2}$ and the gyrostat $S_{0}$ is oblate, then there exists an unique 1-parametric family of relative equilibria of Euler type; on the other hand, if the gyrostat $S_{0}$ is prolate and we have:

(1) $\beta_{1}<R_{1}\left(\xi_{1}\right)$, then there exists an unique 1-parametric family of relative equilibria of Euler type.

(2) $\beta_{1}=R_{1}\left(\xi_{1}\right)$, then two relative equilibria of Euler type exist.

(3) $R_{1}\left(\xi_{1}\right)<\beta_{1}<0$, then three 1-parametric families of relative equilibria of Euler type exist. If $m_{1}=m_{2}$ and $S_{0}$ is oblate, then relative equilibria of Euler type do not exist; but if $S_{0}$ is prolate we have:

(4) $R_{1}(-1 / 2)<\beta_{1}<0$, then two 1 -parametric families of relative equilibria of Euler type exist.

(5) $\beta_{1}=R_{1}(-1 / 2)$, there exists an only equilibrium of Euler type.

(6) $\beta_{1}<R_{1}(-1 / 2)$, then relative equilibria of Euler type do not exist.

The results for the configuration $S_{2} S_{1} S_{0}$ are similar to that of the configuration $S_{0} S_{2} S_{1}$.

4.1.1. Number of Eulerian equilibria in an approximate dynamics of order $k$. In the approximate dynamics of order $k$, the polynomial $p_{k}(\rho)$, that determines the Eulerian equilibria has degree $5+4 k$. Similar results to the previous ones show

$$
p_{k}(\rho)=m_{0} a^{2} \rho^{2}(\rho+1)^{2} p_{k-1}(\rho)+\beta_{k} q_{k-1}(\rho)
$$

with $q_{k-1}(\rho)$ being a positive polynomial. In general, for usual celestial bodies, $\beta_{k} \approx 0$ for $k>1$. Using a recurrent reasoning and applying the implicit function theorem, the number of Eulerian equilibria in the approximate dynamics of order $k$ is the same as that of the approximate dynamics of order one. If $\beta_{k}$ is not close to zero, for certain $k$, a particular analysis of the equation $p_{k}(\rho)$ should be made.

4.2. Stability of Eulerian relative equilibria. The tangent flow of (2.7) in the equilibrium $\mathbf{z}_{e}$ comes given by

$$
\frac{d \delta \mathbf{z}}{d t}=\mathfrak{U}\left(\mathbf{z}_{e}\right) \delta \mathbf{z}
$$

with $\delta \mathbf{z}=\mathbf{z}-\mathbf{z}_{e}$ and $\mathfrak{U}\left(\mathbf{z}_{e}\right)$ is the Jacobian matrix of (2.7) in $\mathbf{z}_{e}$. 
The characteristic polynomial $\mathfrak{U}\left(\mathbf{z}_{e}\right)$ has the following expression:

$$
p=\lambda\left(\lambda^{2}+\Phi^{2}\right)\left(\lambda^{4}+m \lambda^{2}+n\right)\left(\lambda^{8}+p \lambda^{6}+q \lambda^{4}+r \lambda^{2}+s\right)
$$

with $\Phi=\left((C-A) \omega_{e}+l\right) / A$, where the coefficients that intervene in the previous polynomial are functions of the parameters of the problem and $\rho$ being $\rho$ the root of (3.10).

4.2.1. Order-zero approximate dynamics. The characteristic polynomial (4.16) of $\mathfrak{U}\left(\mathbf{z}_{e}\right)$ simplifies to

$$
p=\lambda^{3}\left(\lambda^{2}+\Phi^{2}\right)\left(\lambda^{2}+\omega_{e}^{2}\right)^{2}\left(\lambda^{2}+p\right)\left(\lambda^{4}+q \lambda^{2}+r\right)
$$

with coefficients expressed in Appendix B.

If $p \geq 0, q \geq 0, r \geq 0, q^{2}-4 r \geq 0$, then $\mathbf{z}_{e}$ is spectrally stable. These conditions are not verified since $r<0$.

Proposition 4.5. If $\mathbf{z}_{e}$ is the only relative equilibrium in the configuration $S_{0} S_{2} S_{1}$ of the zero-order approximate dynamics, then this is unstable.

4.2.2. Order-one approximate dynamics. We will analyze the case in which the gyrostat is close to sphere. In this case $C-A \approx 0$, then applying the implicit function theorem, $\mathbf{z}_{e}$ is unstable.

If $C-A$ is not close to zero, the coefficients of the polynomial (4.16) have very complicated expressions. Numeric calculations prove that there exist, for certain values of the parameter $C-A$, linear stable Eulerian relative equilibria (see Vera [5] for details).

These results are equally valid for the configurations $S_{2} S_{0} S_{1}$ and $S_{2} S_{1} S_{0}$.

\section{Conclusions and future works}

The approximate Poisson dynamics of a gyrostat (or rigid body) in Newtonian interaction with two spherical or punctual rigid bodies is considered. We give global conditions on the existence of Eulerian equilibria and in analogy with classic results on the topic, we study the existence of equilibria that we denominate of Euler type in the case in which $S_{1}, S_{2}$ are spherical or punctual bodies and $S_{0}$ is a gyrostat. Necessary and sufficient conditions for their existence in a approximate dynamics of order $k$ are obtained and we give explicit expressions of these equilibria, useful for the later study of the stability of the same ones. A complete study of the number of Eulerian equilibria is made in approximate dynamics of orders zero and one. The number of Eulerian equilibria in an approximate dynamics of order $k$ for $k>1$ is independent of the order of truncation of the potential if the gyrostat $S_{0}$ is close to the sphere. The instability of Eulerian equilibria is proven for any approximate dynamics if the gyrostat is close to the sphere

The methods employed in this work are susceptible of being used in similar problems. Numerous problems are open, and among them it is necessary to consider the study of the possible existence of the "inclined" relative equilibria, in which $\boldsymbol{\Omega}_{e}$ form an angle $\alpha \neq 0$ and $\pi / 2$ with the vector $\lambda^{e}$. 


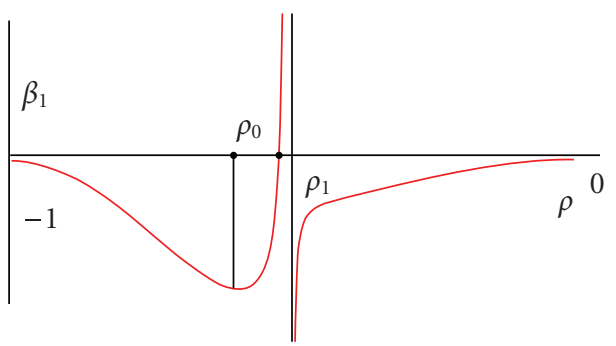

Figure A.1. Function $R_{1}(\rho)$ for $m_{1} \neq m_{2}$.

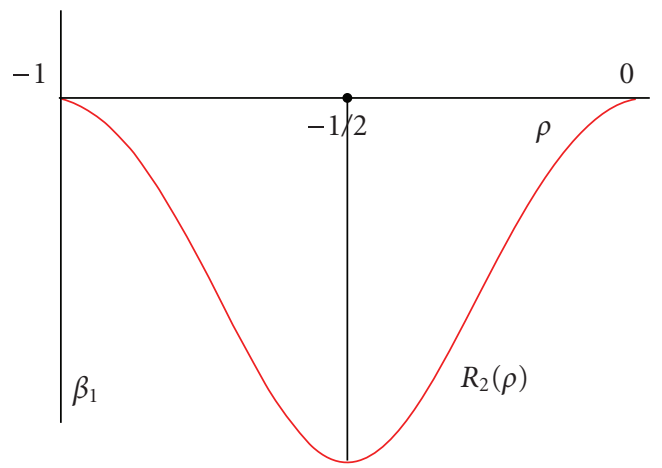

Figure A.2. Function $R_{1}(\rho)$ for $m_{1}=m_{2}$.

\section{Appendices}

A. The function $R_{1}(\rho)$ in approximate dynamics of order one for the configuration $S_{2} S_{0} S_{1}$

B. Coefficients of the characteristic polynomial in Eulerian relative equilibria

The coefficients of the characteristic polynomial (4.17) are

$$
\begin{gathered}
\omega_{e}^{2}=\frac{G\left(\left(m_{2}+m_{1}\right) \rho^{4}+\left(2 m_{1}+2 m_{2}\right) \rho^{3}+\left(m_{2}+m_{1}\right) \rho^{2}-2 m_{0} \rho-m_{0}\right)}{\lambda_{e}^{3}(1+\rho)^{2} \rho^{2}}, \\
\begin{aligned}
p=\frac{G\left(\left(m_{2}+4 m_{0}+m_{1}\right) \rho^{3}+\left(3 m_{2}+6 m_{0}\right) \rho^{2}+\left(4 m_{0}+3 m_{2}\right) \rho+m_{0}+m_{2}\right)}{(1+\rho)^{3} \rho^{3} \lambda_{e}^{3}}, \\
q=G\left(\left(-2 m_{1} \rho^{4} m_{2}+\left(-2 m_{0} m_{1}+m_{1}^{2}+m_{2}^{2}-2 m_{1} m_{2}-2 m_{0} m_{2}\right) \rho^{3}\right.\right. \\
\quad+\left(3 m_{2}^{2}+m_{1} m_{2}-6 m_{0} m_{1}\right) \rho^{2}+\left(-m_{1} m_{2}+3 m_{2}^{2}+2 m_{0} m_{2}-4 m_{0} m_{1}\right) \rho \\
\left.\left.\quad+m_{2}^{2}-m_{0} m_{1}+m_{0} m_{2}-m_{1} m_{2}\right)\right) /\left((1+\rho)^{3} \rho^{3} \lambda_{e}^{3}\right), \\
r=\frac{G^{2}\left(a_{1} \rho^{4}+a_{2} \rho^{4}+a_{3} \rho^{2}+a_{4} \rho+a_{5}\right)}{\left((1+\rho)^{8} \rho^{8} \lambda_{e}^{9}\right)} .
\end{aligned}
\end{gathered}
$$


16 Eulerian equilibria for a gyrostat in the three-body problem

B.1. Coefficients $a_{i}(i=1, \ldots, 5)$

$$
\begin{aligned}
& a_{1}=-42 m_{2}^{7} m_{1}-48 m_{2}^{7} m_{0}-147 m_{2}^{6} m_{1}^{2}-336 m_{2}^{6} m_{1} m_{0}-129 m_{2}^{6} m_{0}^{2} \\
& -207 m_{2}^{5} m_{1}^{3}-782 m_{2}^{5} m_{1}^{2} m_{0}-673 m_{2}^{5} m_{1} m_{0}^{2}-81 m_{2}^{5} m_{0}^{3}-150 m_{2}^{4} m_{1}^{4} \\
& -869 m_{2}^{4} m_{1}^{3} m_{0}-1325 m_{2}^{4} m_{1}^{2} m_{0}^{2}-378 m_{2}^{4} m_{1} m_{0}^{4}-64 m_{2}^{3} m_{1}^{5} \\
& -513 m_{2}^{3} m_{1}^{4} m_{0}-1270 m_{2}^{3} m_{1}^{3} m_{0}^{2}-702 m_{2}^{3} m_{1}^{2} m_{0}^{3}-14 m_{2}^{2} m_{1}^{6} \\
& -165 m_{2}^{2} m_{1}^{5} m_{0}-610 m_{2}^{2} m_{1}^{4} m_{0}^{2}-648 m_{2}^{2} m_{1}^{3} m_{0}^{3}-24 m_{2} m_{1}^{6} m_{0} \\
& -119 m_{2} m_{1}^{5} m_{0}^{2}-297 m_{2} m_{1}^{4} m_{0}^{3}+2 m_{1}^{6} m_{0}^{2}-54 m_{1}^{5} m_{0}^{3} \text {. } \\
& a_{2}=-60 m_{2}^{7} m_{1}-54 m_{2}^{7} m_{0}-243 m_{2}^{6} m_{1}^{2}-474 m_{2}^{6} m_{1} m_{0}-173 m_{2}^{6} m_{0}^{2} \\
& -399 m_{2}^{5} m_{1}^{3}-1345 m_{2}^{5} m_{1}^{2} m_{0}-999 m_{2}^{5} m_{1} m_{0}^{2}-135 m_{2}^{5} m_{0}^{3}-329 m_{2}^{4} m_{1}^{4} \\
& -1846 m_{2}^{4} m_{1}^{3} m_{0}-2223 m_{2}^{4} m_{1}^{2} m_{0}^{2}-648 m_{2}^{4} m_{1} m_{0}^{3}-138 m_{2}^{3} m_{1}^{5} \\
& -1364 m_{2}^{3} m_{1}^{4} m_{0}-2506 m_{2}^{3} m_{1}^{3} m_{0}^{2}-1242 m_{2}^{3} m_{1}^{2} m_{0}^{3}-24 m_{2}^{2} m_{1}^{6} \\
& -536 m_{2}^{2} m_{1}^{5} m_{0}-1530 m_{2}^{2} m_{1}^{4} m_{0}^{2}-1188 m_{2}^{2} m_{1}^{3} m_{0}^{3}-90 m_{2} m_{1}^{6} m_{0} \\
& -477 m_{2} m_{1}^{5} m_{0}^{2}-567 m_{2} m_{1}^{4} m_{0}^{3}-56 m_{1}^{6} m_{0}^{2}-108 m_{1}^{5} m_{0}^{3} \text {. } \\
& a_{3}=-42 m_{2}^{7} m_{1}-36 m_{2}^{7} m_{0}-183 m_{2}^{6} m_{1}^{2}-342 m_{2}^{6} m_{1} m_{0}-93 m_{2}^{6} m_{0}^{2} \\
& -349 m_{2}^{5} m_{1}^{3}-1097 m_{2}^{5} m_{1}^{2} m_{0}-630 m_{2}^{5} m_{1} m_{0}^{2}-81 m_{2}^{5} m_{0}^{3}-358 m_{2}^{4} m_{1}^{4} \\
& -1776 m_{2}^{4} m_{1}^{3} m_{0}-166 m_{2}^{4} m_{1}^{2} m_{0}^{2}-405 m_{2}^{4} m_{1} m_{0}^{3}-189 m_{2}^{3} m_{1}^{5} \\
& -1614 m_{2}^{3} m_{1}^{4} m_{0}-2256 m_{2}^{3} m_{1}^{3} m_{0}^{2}-810 m_{2}^{3} m_{1}^{2} m_{0}^{3}-31 m_{2}^{2} m_{1}^{6} \\
& -827 m_{2}^{2} m_{1}^{5} m_{0}-1683 m_{2}^{2} m_{1}^{4} m_{0}^{2}-810 m_{2}^{2} m_{1}^{3} m_{0}^{3}-6 m_{2} m_{1}^{7} \\
& -228 m_{2} m_{1}^{6} m_{0}-666 m_{2} m_{1}^{5} m_{0}^{2}-405 m_{2} m_{1}^{4} m_{0}^{3}-30 m_{1}^{7} m_{0} \\
& -81 m_{1}^{5} m_{0}^{3}-111 m_{1}^{6} m_{0}^{2} \text {. } \\
& a_{4}=-12 m_{2}^{7} m_{1}-12 m_{2}^{7} m_{0}-56 m_{2}^{6} m_{1}^{2}-114 m_{2}^{6} m_{1} m_{0}-24 m_{2}^{6} m_{0}^{2} \\
& -130 m_{2}^{5} m_{1}^{3}-387 m_{2}^{5} m_{1}^{2} m_{0}-162 m_{2}^{5} m_{1} m_{0}^{2}-179 m_{2}^{4} m_{1}^{4} \\
& -687 m_{2}^{4} m_{1}^{3} m_{0}-432 m_{2}^{4} m_{1}^{2} m_{0}^{2}-140 m_{2}^{3} m_{1}^{5}-693 m_{2}^{3} m_{1}^{4} m_{0} \\
& -588 m_{2}^{3} m_{1}^{3} m_{0}^{2}-52 m_{2}^{2} m_{1}^{6}-387 m_{2}^{2} m_{1}^{5} m_{0}-432 m_{2}^{2} m_{1}^{4} m_{0}^{2}-6 m_{2} m_{1}^{7} \\
& -108 m_{2} m_{1}^{6} m_{0}-162 m_{2} m_{1}^{5} m_{0}^{2}-12 m_{1}^{7} m_{0}-24 m_{1}^{6} m_{0}^{2} \text {. } \\
& a_{5}=-\left(m_{0}+m_{2}\right)\left(18 m_{0} m_{2}^{6}+12 m_{1} m_{2}^{6}+94 m_{2}^{5} m_{0} m_{1}+36 m_{1}^{2} m_{2}^{5}\right. \\
& +81 m_{2}^{4} m_{0}^{2} m_{1}+168 m_{2}^{4} m_{0} m_{1}^{2}+42 m_{2}^{4} m_{1}^{3}+128 m_{2}^{3} m_{0} m_{1}^{3} \\
& +27 m_{2}^{3} m_{1}^{4}+15 m_{2}^{2} m_{1}^{5}+31 m_{2}^{2} m_{0} m_{1}^{4}+126 m_{2}^{2} m_{0}^{2} m_{1}^{3}+18 m_{0}^{2} m_{2}^{5} \\
& +54 m_{2} m_{0}^{2} m_{1}^{4}+12 m_{2} m_{0} m_{1}^{5}+5 m_{2} m_{1}^{6}+7 m_{1}^{6} m_{0}+9 m_{0}^{2} m_{1}^{5} \\
& \left.+144 m_{2}^{3} m_{0}^{2} m_{1}^{2}\right) \text {. }
\end{aligned}
$$




\section{Acknowledgments}

The authors are grateful to the referee for his useful suggestions and comments which improved the paper. This research was partially supported by the Spanish Ministerio de Ciencia y Tecnología (Project BFM2003-02137) and by the Consejería de Educación y Cultura de la Comunidad Autónoma de la Región de Murcia (Project Séneca 2002: PC$\mathrm{MC} / 3 / 00074 / \mathrm{FS} / 02)$.

\section{References}

[1] G. N. Doubochine, On the problem of three rigid bodies, Celestial Mechanics \& Dynamical Astronomy 33 (1984), no. 1, 31-47.

[2] E. Leimanis, The General Problem of the Motion of Coupled Rigid Bodies about a Fixed Point, Springer, Berlin, 1965.

[3] A. J. Maciejewski, Reduction, relative equilibria and potential in the two rigid bodies problem, Celestial Mechanics \& Dynamical Astronomy 63 (1995), no. 1, 1-28.

[4] F. Mondejar and A. Vigueras, The Hamiltonian dynamics of the two gyrostats problem, Celestial Mechanics \& Dynamical Astronomy 73 (1999), no. 1-4, 303-312.

[5] J. A. Vera, Reducciones, equilibrios y estabilidad en dinámica de sólidos rígidos y giróstatos, Ph.D. dissertation, Universidad Politécnica de Cartagena, Cartagena, 2004.

[6] J. A. Vera and A. Vigueras, Hamiltonian dynamics of a gyrostat in the n-body problem: relative equilibria, Celestial Mechanics \& Dynamical Astronomy 94 (2006), no. 3, 289-315.

[7] V. V. Vidyakin, Euler solutions in the problem of translational-rotational motion of three-rigid bodies, Celestial Mechanics \& Dynamical Astronomy 16 (1977), no. 4, 509-526.

[8] L. S. Wang, P. S. Krishnaprasad, and J. H. Maddocks, Hamiltonian dynamics of a rigid body in a central gravitational field, Celestial Mechanics \& Dynamical Astronomy 50 (1991), no. 4, 349386.

[9] S. G. Zhuravlev and A. A. Petrutskii, Current state of the problem of translational-rotational motion of three rigid bodies, Soviet Astronomy 34 (1990), no. 3, 299-304.

J. A. Vera: Departamento de Matemática Aplicada y Estadística, Universidad Politécnica de Cartagena, 30203 Cartagena, Murcia, Spain

E-mail addresses: juanantonio.vera@upct.es; juanantonia.vera@educarm.es

A. Vigueras: Departamento de Matemática Aplicada y Estadística, Universidad Politécnica de Cartagena, 30203 Cartagena, Murcia, Spain

E-mail address: antonio.vigueras@upct.es 


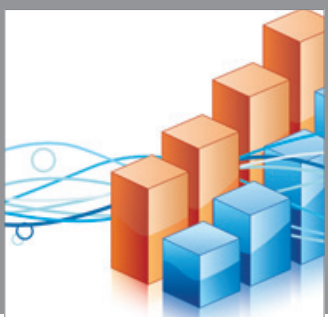

Advances in

Operations Research

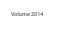

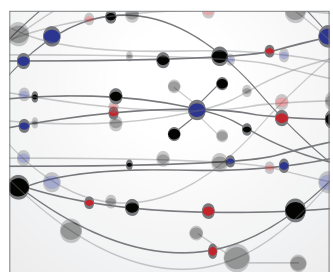

\section{The Scientific} World Journal
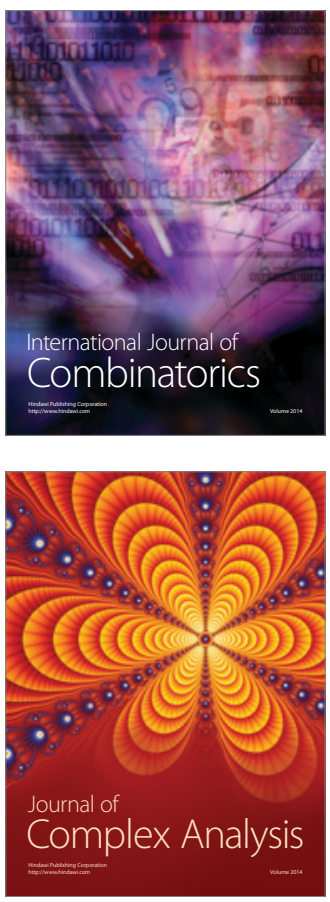

International Journal of

Mathematics and

Mathematical

Sciences
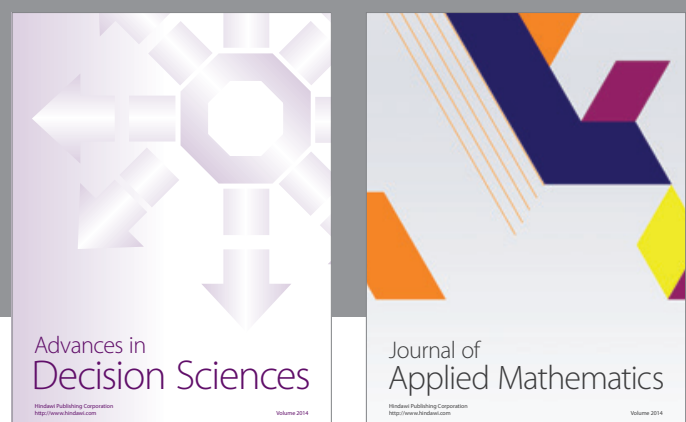

Journal of

Applied Mathematics
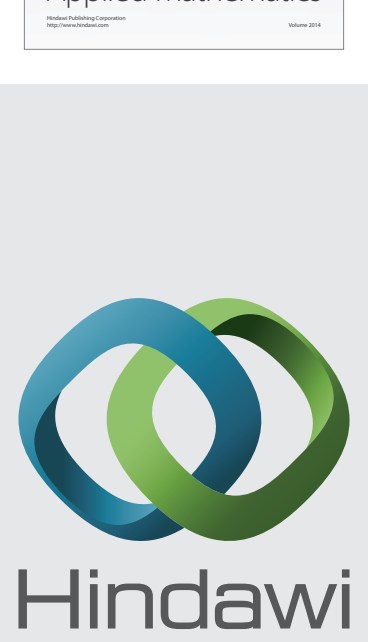

Submit your manuscripts at http://www.hindawi.com
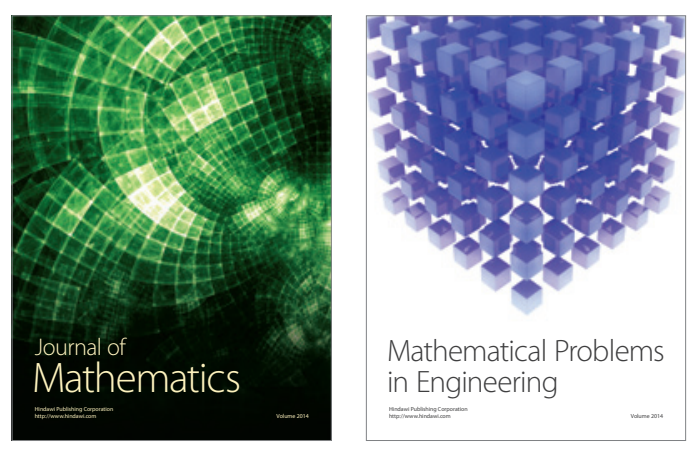

Mathematical Problems in Engineering
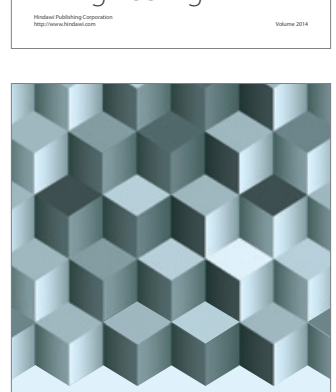

Journal of

Function Spaces
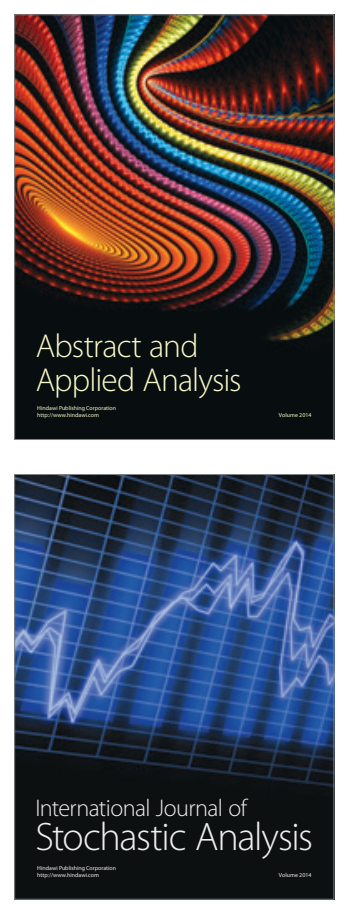

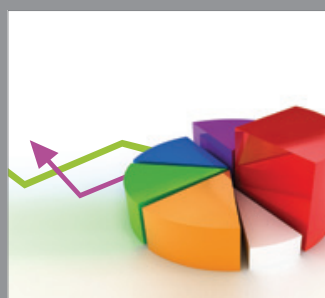

ournal of

Probability and Statistics

Promensencen
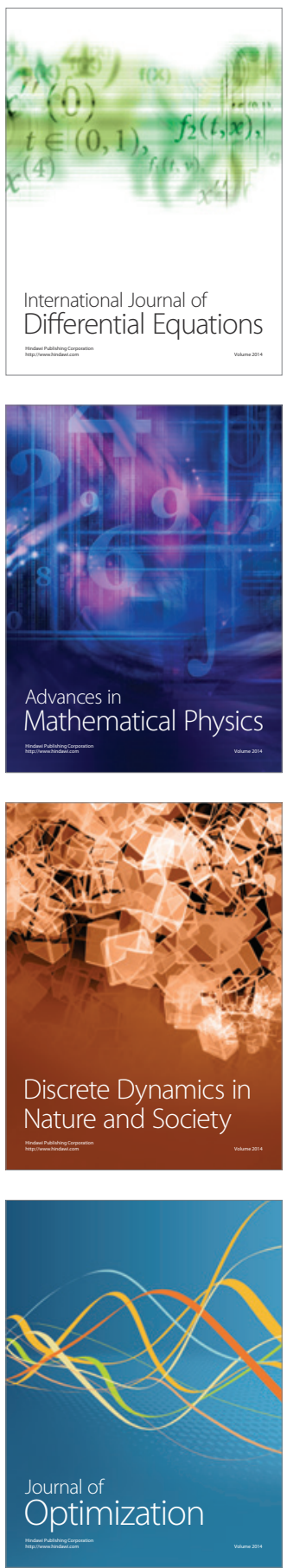